\title{
Boundary layer effects on ionic flows via classical Poisson-Nernst-Planck systems
}

https://doi.org/10.1515/cmb-2018-0002

Received June 30, 2018; accepted August 30, 2018

Abstract: A quasi-one-dimensional steady-state Poisson-Nernst-Planck model of two oppositely charged ion species through a membrane channel is analyzed. The model problem is treated as a boundary value problem of a singularly perturbed differential system. Our analysis is based on the geometric singular perturbation theory but, most importantly, on specific structures of this concrete model. The existence and (local ) uniqueness of solutions to the boundary value problem is established. In particular, an approximation of both the individual flux and the I-V (current-voltage) relation are derived explicitly from the zeroth order approximation (in $\varepsilon$ ) solutions, from which the boundary layer effects on ionic flows are studied in great details.

Keywords: Poisson-Nernst-Planck systems, boundary layers, individual fluxes, total flux

MSC: 34A26, 34B16, 34D15, 37D10, 92C35

\section{Introduction}

One of the fundamental concerns of physiology is the function of ion channels. Ion channels are approximately cylindrical, hollow proteins with a hole down their middle that provides a controllable path for electrodiffusion of ions (mainly $\mathrm{Na}^{+}, \mathrm{K}^{+}, \mathrm{Ca}^{++}$and $\mathrm{Cl}^{-}$) through biological membranes, establishing communications among cells and the external environment. In this way, ion channels control a wide range of biological functions. In biological devices, macroscopic flows of charges are driven through tiny (atomic scale) channels that link one macroscopic reservoir to another. The reservoirs are macroscopic regions in which the concentration of charges is nearly constant (because the dimensions of the reservoirs are macroscopic and so the total number of charges is hardly changed by the flows) and electrical potentials are nearly constant too. To describe the actual behavior of channels (or useful transistors) (see [7, 22-24, 47-50], etc.), macroscopic reservoirs must be included in the mathematical formulation. Macroscopic boundary conditions that describe such reservoirs introduce boundary layers of concentration and charge. It is very interesting and worthy to analyze the effects on ionic flows from the boundary layers. Ionic flows are governed by fundamental physical laws of electrodiffusion which relate rates of quantities of interest. The macroscopic properties of ionic flows through ion channels with given structures rely further on external driving forces, namely, boundary potentials and concentrations of ion species involved. Two most relevant biological properties of a channel are permeation and selectivity, both of which are characterized by the current-voltage (I-V) relations measured experimentally under different ionic conditions.

Taking the structural characteristics into consideration, the basic continuum model for ionic flows is the Poisson-Nernst-Planck (PNP) system, which treats the aqueous medium (within which ions are migrating) as a dielectric continuum (see [7, 8, 10, 12, 14-19, 22-25, 30, 31, 38, 54], etc.). Under some reasonable conditions, the PNP system can be derived from more fundamental models such as the Langevin-Poisson system (see, for

^Corresponding Author: Mingji Zhang: Department of Mathematics, New Mexico Institute of Mining and Technology, Socorro, NM 87801, USA, E-mail: mingji.zhang@nmt.edu, mzhang0129@gmail.com. 
example, [13, 31, 46, 52, 54, 55]) or the Maxwell-Boltzmann equations (see, for example, [2, 30, 31, 54]), and from an energy variational analysis (see [27-29, 60, 63]).

The simplest PNP model is the classical PNP (cPNP) system that contains only the ideal component of electrochemical potential. The cPNP system treats ions essentially as point-charges, and neglects ion size effects. It has been simulated (see, e.g., $[9,10,12,14,22,26])$ and analyzed (see, e.g., $[1,3-5,20,21,33,39$, $41-43,53,56,57,59,61])$ to a great extent. Very often, the so-called electroneutrality boundary conditions are enforced at both ends of the channel in the study (see, e.g., [5, 6, 32, 33, 40-42, 45, 61]). This greatly reduces the difficulty in mathematical analysis of qualitative properties of ionic flows because, under the assumption of electroneutrality boundary concentrations, the boundary layers disappear in the study of PNP model for membrane channels (see, e.g., $[1,6,33,34,40,62]$ ). Therefore, to consider the boundary layer effects on ionic flows, one should remove the neutral conditions on boundary concentrations. However, if those boundary layers reach into the part of the device performing atomic control, they dramatically affect its behavior. Boundary layers of charge are particularly likely to create artifacts over long distances because the electric field spreads a long way. Therefore, a first step to analyze boundary layer effects on ionic flows is to study the state that is not neutral but close, which is our main focus in this work.

The rest of the paper is organized as follows. In Section 2, we describe the quasi-one-dimensional PNP model of ion flows, the formulation of the boundary value problem of the singularly perturbed PNP system, and the basic assumptions. In Section 3, we cast our problem in the language of geometric singular perturbation theory. Upon introducing new dependent variables, we rewrite the PNP system as a standard singularly perturbed system of first order differential equations. Making use of the inner and outer limiting systems, we construct a singular orbit for the PNP boundary value problem. We then apply geometric singular perturbation theory, such as Exchange Lemma, to show that, for small $\varepsilon>0$, there is a true solution shadowing the singular orbit. Our main results are stated in Section 4. In this part, without the assumption of electroneutrality conditions, we study the boundary layer effects on ionic flows in terms of both the individual flux and the total flux of charge. Some critical potentials are identified, which play a key role in characterizing the effect from boundary layers, more precisely, from the violation of electroneutrality boundary conditions. It also turns out that the effect depends sensitively on multiple physical parameters such as boundary concentrations, ion valence and diffusion coefficients. The paper ends with a concluding remark provided in Section 5.

\section{Models and assumptions}

In this section, we briefly recall the model of Poisson-Nernst-Planck (PNP) systems, and set up our problem with some concrete assumptions.

We assume the channel is narrow so that it can be effectively viewed as a one-dimensional channel that connects the interior and the exterior of the channel. A quasi-one-dimensional steady-state PNP model for ion flows of $n$ ion species though a single channel is (see $[44,51]$ )

$$
\begin{aligned}
& \frac{1}{A(X)} \frac{d}{d X}\left(\varepsilon_{r}(X) \varepsilon_{0} A(X) \frac{d \Phi}{d X}\right)=-e\left(\sum_{j=1}^{n} z_{j} C_{j}(X)+Q(X)\right), \\
& \frac{d \mathcal{J}_{i}}{d X}=0, \quad-\mathcal{J}_{i}=\frac{1}{k_{B} T} \mathcal{D}_{i}(X) A(X) C_{i}(X) \frac{d \mu_{i}}{d X}, \quad i=1,2, \cdots, n,
\end{aligned}
$$

where $X \in[0, l], e$ is the elementary charge, $k_{B}$ is the Boltzmann constant, $T$ is the absolute temperature; $\Phi$ is the electric potential, $Q(X)$ is the permanent charge of the channel, $\varepsilon_{r}(X)$ is the relative dielectric coefficient, $\varepsilon_{0}$ is the vacuum permittivity; $A(X)$ is the area of the cross-section of the channel over the point $X \in[0, l]$; for the $i$ th ion species, $C_{i}$ is the concentration (number of $i$ th ions per volume), $z_{i}$ is the valence (number of charges per particle) that is positive for cations and negative for anions, $\mu_{i}$ is the electrochemical potential, $\mathcal{J}_{i}$ is the flux density, and $\mathcal{D}_{i}(X)$ is the diffusion coefficient. 
For system (2.1), we impose the following boundary conditions (see, [20] for justification), for $k=$ $1,2, \cdots, n$,

$$
\Phi(0)=V, \quad C_{i}(0)=\mathcal{L}_{i}>0 ; \quad \Phi(l)=0, \quad C_{i}(l)=\mathcal{R}_{i}>0
$$

For ion channels, an important characteristic is the so-called I-V relations (current-voltage relations). For a solution of the steady-state boundary value problem (2.1)-(2.2), the rate of flow of charge through a crosssection or current $\mathcal{J}$ is

$$
\mathcal{J}=\sum_{j=1}^{n} z_{j} \mathcal{J}_{j} .
$$

For fixed boundary concentrations $\mathcal{L}_{i}$ 's and $\mathcal{R}_{i}$ 's, $\mathcal{J}_{j}$ 's depend on $\bar{V}$ only and formula (2.3) provides a relation of the current $\mathcal{J}$ on the voltage $\bar{V}$. This relation is the I-V relation.

\subsection{The steady-state boundary value problem and assumptions}

The main goal of this paper is to examine the boundary layers effects on ionic flows via the steady-state PNP system (2.1)-(2.2). For definiteness, we will take the following setting:

(A1). We consider two ion species $(n=2)$ with $z_{1}>0$ and $z_{2}<0$.

(A2). We assume the permanent charge $Q(X)$ to be zero.

(A3). For the electrochemical potential $\mu_{i}$, only the ideal component $\mu_{i}^{i d}(X)$ is included, which is defined by

$$
\mu_{i}^{i d}(X)=z_{i} e \Phi(X)+k_{B} T \ln \frac{C_{i}(X)}{C_{0}}
$$

(A4). The relative dielectric coefficient and the diffusion coefficient are constants, that is, $\varepsilon_{r}(X)=\varepsilon_{r}$ and $D_{i}(X)=D_{i}$.

In the sequel, we will assume (A1)-(A4). We first make a dimensionless rescaling following ([21]). Set $C_{0}=\max \left\{\mathcal{L}_{i}, \mathcal{R}_{i}: i=1,2\right\}$ and let

$$
\begin{aligned}
\varepsilon^{2} & =\frac{\varepsilon_{r} \varepsilon_{0} k_{B} T}{e^{2} l^{2} C_{0}}, \quad x=\frac{X}{l}, \quad h(x)=\frac{A(X)}{l^{2}}, \quad D_{i}=l C_{0} \mathcal{D}_{i} ; \\
\phi(x) & =\frac{e}{k_{B} T} \Phi(X), \quad c_{i}(x)=\frac{C_{i}(X)}{C_{0}}, \quad J_{i}=\frac{\partial_{i}}{D_{i}} ; \\
\bar{V} & =\frac{k_{B} T}{e} V, \quad L_{i}=\frac{\mathcal{L}_{i}}{C_{0}} ; \quad R_{i}=\frac{\mathcal{R}_{i}}{C_{0}} .
\end{aligned}
$$

Using expressions (2.4) for the ideal component $\mu_{i}^{i d}(X)$, the boundary value problem (2.1)-(2.2) becomes

$$
\begin{gathered}
\varepsilon^{2} h^{-1}(x) \frac{d}{d x}\left(h(x) \frac{d}{d x} \phi\right)=-\left(z_{1} c_{1}+z_{2} c_{2}\right), \quad \frac{d J_{i}}{d x}=0, \\
\frac{d c_{1}}{d x}=-z_{1} c_{1} \frac{d \phi}{d x}-\frac{J_{1}}{h(x)}, \quad \frac{d c_{2}}{d x}=-z_{2} c_{2} \frac{d \phi}{d x}-\frac{J_{2}}{h(x)},
\end{gathered}
$$

with boundary conditions, for $i=1,2$,

$$
\phi(0)=\bar{V}, \quad c_{i}(0)=L_{i} ; \phi(1)=0, \quad c_{i}(1)=R_{i} .
$$

Remark 2.1. The dimensionless parameter $\varepsilon$ defined in (2.5) as $\varepsilon=\frac{1}{l} \sqrt{\frac{\varepsilon_{r} \varepsilon_{0} k_{B} T}{e^{2} C_{0}}}$ is directly related to the ratio $\kappa_{D} / l$, where $\kappa_{D}=\sqrt{\frac{\varepsilon_{r} \varepsilon_{0} k_{B} T}{\sum_{j}\left(z_{j} e\right)^{2} C_{j}}}$ is the Debye length; in particular, $\varepsilon=\kappa_{D} / l$ when $z_{j}^{2}=1$ and $C_{j}=C_{0}$. Typically, the parameter $\varepsilon$ is small due to the fact that the two variables $l$, the length of the channel, and $C_{0}$, some characteristic number density could be very large ( for many cases, the value of $\varepsilon$ is of order $O\left(10^{-3}\right)$ ). One may also refer to [11] for more information related to the dimensionless parameter $\varepsilon$. 


\section{Geometric singular perturbation theory for (2.6)-(2.7)}

We first rewrite system (2.6) into a standard form for singularly perturbed systems and convert the boundary value problem (2.6)-(2.7) to a connecting problem.

Denote the derivative with respect to $x$ by overdot and introduce $u=\varepsilon \dot{\phi}$ and $\tau=x$. System (2.6) becomes

$$
\begin{aligned}
& \varepsilon \dot{\phi}=u, \quad \varepsilon \dot{u}=-z c_{1}-z c_{2}-\varepsilon \frac{h^{\prime}(\tau)}{h(\tau)} u, \quad \varepsilon \dot{c}_{1}=-z c_{1} u-\varepsilon h^{-1}(\tau) J_{1}, \\
& \varepsilon \dot{c}_{2}=-z c_{2} u-\varepsilon h^{-1}(\tau) J_{2}, \quad \dot{J}_{k}=0, \quad \dot{\tau}=1 .
\end{aligned}
$$

System (3.1), the so-called slow system, will be treated as a singularly perturbed system with $\varepsilon$ as the singular parameter. Its phase space is $\mathbb{R}^{7}$ with state variables $\left(\phi, u, c_{1}, c_{2}, J_{1}, J_{2}, \tau\right)$.

For $\varepsilon>0$, the rescaling $x=\varepsilon \xi$ of the independent variable gives rise to the so-called fast system

$$
\begin{aligned}
& \phi^{\prime}=u, \quad u^{\prime}=-z c_{1}-z c_{2}-\varepsilon \frac{h^{\prime}(\tau)}{h(\tau)} u, \quad c_{1}^{\prime}=-z c_{1} u-\varepsilon h^{-1}(\tau) J_{1}, \\
& c_{2}^{\prime}=-z c_{2} u-\varepsilon h^{-1}(\tau) J_{2}, \quad J_{k}^{\prime}=0, \quad \tau^{\prime}=\varepsilon,
\end{aligned}
$$

where prime denotes the derivative with respect to the variable $\xi$.

For $\varepsilon>0$, slow system (3.1) and fast system (3.2) have exactly the same phase portrait. But their limiting systems at $\varepsilon=0$ are different. The limiting system of (3.1) is called the limiting slow system, whose orbits are called slow orbits or regular layers. The limiting system of (3.2) is the limiting fast system, whose orbits are called fast orbits or singular (boundary and/or internal) layers. In this context, a singular orbit of system (3.1) or (3.2) is defined to be a continuous and piecewise smooth curve in $\mathbb{R}^{7}$ that is a union of finitely many slow and fast orbits. Very often, limiting slow and fast systems provide complementary information on state variables. Therefore, the main task is to patch the limiting information together to form a solution for the entire $\varepsilon>0$ system.

Let $B_{L}$ and $B_{R}$ be the subsets of the phase space $\mathbb{R}^{7}$ defined by

$$
\begin{aligned}
& B_{L}=\left\{\left(\bar{V}, u, L_{1}, L_{2}, J_{1}, J_{2}, 0\right) \in \mathbb{R}^{7}: \operatorname{arbitrary} u, J_{1}, J_{2}\right\}, \\
& B_{R}=\left\{\left(0, u, R_{1}, R_{2}, J_{1}, J_{2}, 1\right) \in \mathbb{R}^{7}: \operatorname{arbitrary} u, J_{1}, J_{2}\right\} .
\end{aligned}
$$

Then the original boundary value problem is equivalent to a connecting problem, namely, finding a solution of (3.1) or (3.2) from $B_{L}$ to $B_{R}$ (see, for example, [35]).

We would like to point out that the existence and uniqueness result of the classical PNP model with $n$ ion species under the framework of geometric singular perturbation theory has been well-established (see e.g., [41, 42]). For completeness and readers' convenience, we briefly describe the process of constructions of singular orbits for this special case with two ion species, one positively charged and one negatively charged. A detailed proof of the existence and uniqueness result of system (3.1) or (3.2) for $\varepsilon>0$ small is provided in this work (see Theorem 3.6).

\subsection{Geometric construction of singular orbits}

We will first construct a singular orbit on $[0,1]$ that connects $B_{L}$ to $B_{R}([20,41,42])$. Such an orbit will generally consist of two boundary layers and a regular layer.

\subsubsection{Limiting fast dynamics and boundary layers}

By setting $\varepsilon=0$ in (3.1), we obtain the so-called slow manifold

$$
z=\left\{u=0, z_{1} c_{1}+z_{2} c_{2}=0\right\} .
$$


By setting $\varepsilon=0$ in (3.2), we get the limiting fast system

$$
\begin{aligned}
& \phi^{\prime}=u, \quad u^{\prime}=-z_{1} c_{1}-z_{2} c_{2}, \quad c_{1}^{\prime}=-z_{1} c_{1} u, \\
& c_{2}^{\prime}=-z_{2} c_{2} u, \quad J_{k}^{\prime}=0, \quad \tau^{\prime}=0 .
\end{aligned}
$$

Note that the slow manifold $z$ is the set of equilibria of (3.5). The following result can be established directly by linearizing the limiting fast system at points from $z$, its equilibrium set ([42]).

Lemma 3.1. For system (3.5), the slow manifold $z$ is normally hyperbolic.

Let $W^{S}(z)$ (resp. $\left.W^{u}(z)\right)$ denote the stable (resp. unstable) manifold of $z$. We denote the collection of orbits from $B_{L}$ in forward time under the flow of system (3.5) by $M_{L}$, and the collection of orbits from $B_{R}$ in backward time under the flow of system (3.5) by $M_{R}$. Then, for a singular orbit connecting $B_{L}$ to $B_{R}$, the boundary layer at $x=0$ must lie in $N_{L}=M_{L} \cap W^{s}(z)$ and the boundary layer at $x=1$ must lie in $N_{R}=M_{R} \cap W^{u}(z)$. In this subsection, we will determine the boundary layers $N_{L}$ and $N_{R}$, and their landing points $\omega\left(N_{L}\right)$ and $\alpha\left(N_{R}\right)$ on the slow manifold $z$. The regular layer, determined by the limiting slow system in §3.1.2, will lie in $z$ and connect the landing points $\omega\left(N_{L}\right)$ at $x=0$ and $\alpha\left(N_{R}\right)$ at $x=1$.

One can easily verify the next result, which is crucial to study the boundary layers of our problem.

Proposition 3.2. The following functions are the first integrals of system (3.5),

$$
\begin{aligned}
& H_{1}=\ln c_{1}+z_{1} \phi, \quad H_{2}=\ln c_{2}+z_{2} \phi, \quad H_{3}=\frac{u^{2}}{2}-c_{1}-c_{2}, \\
& H_{4}=J_{1}, \quad H_{5}=J_{2}, \quad H_{6}=\tau .
\end{aligned}
$$

From Proposition 3.2, together with the definition of first integrals, we have (see also [42])

Proposition 3.3. $\quad$ (i) The stable manifold $W^{s}(Z)$ intersects $B_{L}$ transversally at points

$$
\left(\bar{V}, u^{l}, L_{1}, L_{2}, J_{1}, J_{2}, 0\right),
$$

and the $\omega$-limit set of $N_{L}=M_{L} \cap W^{S}(Z)$ is

$$
\omega\left(N_{L}\right)=\left\{\left(\phi^{L}, 0, c_{1}^{L}, c_{2}^{L}, J_{1}, J_{2}, 0\right)\right\},
$$

where $J_{i}$ for $i=1,2$ are arbitrary, and

$$
\begin{aligned}
\phi^{L} & =\bar{V}-\frac{1}{z_{1}-z_{2}} \ln \frac{-z_{2} L_{2}}{z_{1} L_{1}}, \quad z_{1} c_{1}^{L}=-z_{2} c_{2}^{L}=\left(z_{1} L_{1}\right)^{\frac{-z_{2}}{z_{1}-z_{2}}}\left(-z_{2} L_{2}\right)^{\frac{z_{1}}{z_{1}-z_{2}}}, \\
u^{l} & =\operatorname{sgn}\left(\phi^{L}-\bar{V}\right) \sqrt{2 L_{1}\left(1-e^{z_{1}\left(\bar{V}-\phi^{L}\right)}\right)+2 L_{2}\left(1-e^{z_{2}\left(\bar{V}-\phi^{L}\right)}\right)} .
\end{aligned}
$$

(ii) The unstable manifold $W^{u}(Z)$ intersects $B_{R}$ transversally at points

$$
\left(0, u^{r}, R_{1}, R_{2}, J_{1}, J_{2}, 1\right),
$$

and the $\alpha$-limit set of $N_{R}=M_{R} \cap W^{u}(\mathcal{Z})$ is

$$
\alpha\left(N_{R}\right)=\left\{\left(\phi^{R}, 0, c_{1}^{R}, c_{2}^{R}, J_{1}, J_{2}, 1\right)\right\},
$$

where $J_{i}$ for $i=1,2$ are arbitrary, and

$$
\begin{aligned}
\phi^{R} & =-\frac{1}{z_{1}-z_{2}} \ln \frac{-z_{2} R_{2}}{z_{1} R_{1}}, \quad z_{1} c_{1}^{R}=-z_{2} c_{2}^{R}=\left(z_{1} R_{1}\right)^{\frac{-z_{2}}{z_{1}-z_{2}}}\left(-z_{2} R_{2}\right)^{\frac{z_{1}}{z_{1}-z_{2}}}, \\
u^{r} & =\operatorname{sgn}\left(-\phi^{R}\right) \sqrt{2 R_{1}\left(1-e^{-z_{1} \phi^{R}}\right)+2 R_{2}\left(1-e^{-z_{2} \phi^{R}}\right) .}
\end{aligned}
$$

For later use, let $\Gamma^{0}$ denote the boundary layer at $x=0$ and let $\Gamma^{1}$ denote the boundary layer at $x=1$ for system (3.5). 


\subsubsection{Limiting slow dynamics and regular layers}

Next we construct the regular layer on $z$ that connects $\omega\left(N_{L}\right)$ and $\alpha\left(N_{R}\right)$. Note that, for $\varepsilon=0$, system (3.1) loses most information. To remedy this degeneracy, make a rescaling $u=\varepsilon p$ and $-z_{2} c_{2}=z_{1} c_{1}+\varepsilon q$ in system (3.1) (see also [20, 41, 42]). In term of the new variables, system (3.1) becomes

$$
\begin{aligned}
\dot{\phi} & =p, \quad \varepsilon \dot{p}=q-\varepsilon \frac{h^{\prime}(\tau)}{h(\tau)} p, \\
\varepsilon \dot{q} & =\left(z_{1}\left(z_{1}-z_{2}\right) c_{1}-\varepsilon z_{2} q\right) p+h^{-1}(\tau)\left(z_{1} J_{1}+z_{2} J_{2}\right), \\
\dot{c}_{1} & =-z_{1} c_{1} p-h^{-1}(\tau) J_{1}, \quad \dot{J}_{k}=0, \quad \dot{\tau}=1 .
\end{aligned}
$$

It is again a singular perturbation problem and its limiting slow system is

$$
\begin{aligned}
& \dot{\phi}=p, \quad q=0, \quad p=-\frac{z_{1} J_{1}+z_{2} J_{2}}{z_{1}\left(z_{1}-z_{2}\right) h(\tau) c_{1}}, \\
& \dot{c}_{1}=-z_{1} c_{1} p-J_{1}, \quad \dot{J}_{k}=0, \quad \dot{\tau}=1 .
\end{aligned}
$$

For system (3.7), the slow manifold is

$$
\mathcal{S}=\left\{q=0, p=-\frac{z_{1} J_{1}+z_{2} J_{2}}{z_{1}\left(z_{1}-z_{2}\right) h(\tau) c_{1}}\right\} .
$$

Therefore, the limiting slow system on $\mathcal{S}$ is

$$
\dot{\phi}=-\frac{z_{1} J_{1}+z_{2} J_{2}}{z_{1}\left(z_{1}-z_{2}\right) h(\tau) c_{1}}, \quad \dot{c}_{1}=\frac{z_{2}\left(J_{1}+J_{2}\right)}{\left(z_{1}-z_{2}\right) h(\tau)}, \quad \dot{J}_{k}=0, \quad \dot{\tau}=1 .
$$

Lemma 3.4. There is a unique solution $\left(\phi(x), c_{1}(x), c_{2}(x), J_{1}, J_{2}, J_{3}, \tau(x)\right)$ of (3.8) such that

$$
\left(\phi(0), c_{1}(0), c_{2}(0), \tau(0)\right)=\left(\phi^{L}, c_{1}^{L}, c_{2}^{L}, 0\right) \text { and }\left(\phi(1), c_{1}(1), c_{2}(1), \tau(1)\right)=\left(\phi^{R}, c_{1}^{R}, c_{2}^{R}, 1\right),
$$

where $\phi^{L}, \phi^{R}, c_{1}^{L}, c_{1}^{R}, c_{2}^{L}$ and $c_{2}^{R}$ are given in Proposition 3.3. It is given by

$$
\begin{aligned}
& \phi(x)=\phi^{L}-\frac{z_{1} J_{1}+z_{2} J_{2}}{z_{1} z_{2}\left(J_{1}+J_{2}\right)} \ln \frac{c_{1}(x)}{c_{1}^{L}}, \\
& c_{1}(x)=c_{1}^{L}+\frac{z_{2}\left(J_{1}+J_{2}\right)}{z_{1}-z_{2}} \int_{0}^{x} h^{-1}(s) d s, \quad \tau(x)=x,
\end{aligned}
$$

where $J_{1}$ and $J_{2}$ are uniquely determined as

$$
\begin{aligned}
& J_{1}=\frac{c_{1}^{L}-c_{1}^{R}}{\int_{0}^{1} h^{-1}(s) d s}\left(1+\frac{z_{1}\left(\phi^{L}-\phi^{R}\right)}{\ln c_{1}^{L}-\ln c_{1}^{R}}\right), \\
& J_{2}=\frac{c_{2}^{L}-c_{2}^{R}}{\int_{0}^{1} h^{-1}(s) d s}\left(1+\frac{z_{2}\left(\phi^{L}-\phi^{R}\right)}{\ln c_{2}^{L}-\ln c_{2}^{R}}\right) .
\end{aligned}
$$

The slow orbit

$$
\Lambda(x)=\left(\phi(x), c_{1}(x), c_{2}(x), J_{1}, J_{2}, \tau(x)\right)
$$

given in Lemma 3.4 connects $\omega\left(N_{L}\right)$ and $\alpha\left(N_{R}\right)$. Let $\bar{M}_{L}$ (resp., $\bar{M}_{R}$ ) be the forward (resp., backward) image of $\omega\left(N_{L}\right)$ (resp., $\alpha\left(N_{R}\right)$ ) under the slow flow (3.8). One has the following result whose proof can be established by a similar argument as those in [34, 40, 42].

Proposition 3.5. On the seven-dimensional slow manifold $\mathcal{S}, \bar{M}_{L}$ and $\bar{M}_{R}$ intersect transversally along the unique orbit $\Lambda(x)$ given in (3.11).

To end this section, we would like to point out that the capability of constructing an explicit solution to the limiting slow system, from which the approximated individual flux can be extracted explicitly in terms of boundary conditions and other physical parameters, is crucial for us to further examine the qualitative properties of ionic flows (see Section 4 for details). 


\subsection{Existence of solutions near the singular orbit}

We have constructed a unique singular orbit on $[0,1]$ that connects $B_{L}$ to $B_{R}$. It consists of two boundary layer orbits $\Gamma^{0} \cup \Gamma^{1}$ and a regular layer $\Lambda$ with $\Gamma^{0}$ from the point

$$
\left(\bar{V}, u^{l}, L_{1}, L_{2}, J_{1}, J_{2}, 0\right) \in B_{L} \text { to the point }\left(\phi^{L}, 0, c_{1}^{L}, c_{2}^{L}, J_{1}, J_{2}, 0\right) \in \omega\left(N_{L}\right) \subset z,
$$

and $\Gamma^{1}$ from the point

$$
\left(\phi^{R}, 0, c_{1}^{R}, c_{2}^{R}, J_{1}, J_{2}, 1\right) \in \alpha\left(N_{R}\right) \subset z \text { to the point }\left(0, u^{r}, R_{1}, R_{2}, J_{1}, J_{2}, 1\right) \in B_{R},
$$

and finally $\Lambda \subset Z$ connecting the two landing points

$$
\left(\phi^{L}, 0, c_{1}^{L}, c_{2}^{L}, J_{1}, J_{2}, 0\right) \in \omega\left(N_{L}\right) \text { and }\left(\phi^{R}, 0, c_{1}^{R}, c_{2}^{R}, J_{1}, J_{2}, 1\right) \in \alpha\left(N_{R}\right)
$$

of the two boundary layers.

We now establish the existence of a solution to (2.6)-(2.7) near the singular orbit constructed above which is a union of two boundary layers and one regular layer $\Gamma^{0} \cup \Lambda \cup \Gamma^{1}$. The proof follows the same line as that in $[20,34,40-42]$ and the main tool used is the Exchange Lemma (see, for example [35-37, 58]) of geometric singular perturbation theory.

Theorem 3.6. Let $\Gamma^{0} \cup \Lambda \cup \Gamma^{1}$ be the singular orbit of the connecting problem for (3.1) associated with $B_{L}$ and $B_{R}$ in (3.3). Then, for $\varepsilon>0$ small, the boundary value problem (2.6)-(2.7) has a unique smooth solution near the singular orbit.

Proof. Fix $\delta>0$ small to be determined. Let

$$
B_{L}(\delta)=\left\{\left(\bar{V}, u, L_{1}, L_{2}, J_{1}, J_{2}, 0\right) \in \mathbb{R}^{7}:\left|u-u^{l}\right|<\delta,\left|J_{i}-J_{i}(v)\right|<\delta\right\} .
$$

For $\varepsilon>0$, let $M_{L}(\varepsilon, \delta)$ be the forward trace of $B_{L}(\delta)$ under the flow of system (3.1) or equivalently of system (3.2) and let $M_{R}(\varepsilon)$ be the backward trace of $B_{R}$. To prove the existence and uniqueness statement, it suffices to show that $M_{L}(\varepsilon, \delta)$ intersects $M_{R}(\varepsilon)$ transversally in a neighborhood of the singular orbit $\Gamma^{0} \cup \Lambda \cup \Gamma^{1}$. The latter will be established by an application of the Exchange Lemma.

Notice that $\operatorname{dim} B_{L}(\delta)=3$. It is clear that the vector field of the fast system (3.2) is not tangent to $B_{L}(\delta)$ for $\varepsilon \geq 0$, and hence, $\operatorname{dim} M_{L}(\varepsilon, \delta)=4$. We next apply the Exchange Lemma to track $M_{L}(\varepsilon, \delta)$ in the vicinity of $\Gamma^{0} \cup \Lambda \cup \Gamma^{1}$. First of all, the transversality of the intersection $B_{L}(\delta) \cap W^{s}(Z)$ along $\Gamma^{0}$ in Proposition 3.3 implies the transversality of the intersection $M_{L}(0, \delta) \cap W^{s}(Z)$. Secondly, we have also established that $\operatorname{dim} \omega\left(N_{L}\right)=\operatorname{dim} N_{L}-1=2$ in Proposition 3.3 and that the limiting slow flow is not tangent to $\omega\left(N_{L}\right)$ in Section 3.1.2. Under these conditions, the Exchange Lemma $([35-37,58])$ states that there exist $\rho>0$ and $\varepsilon_{1}>0$ so that, if $0<\varepsilon \leq \varepsilon_{1}$, then $M_{L}(\varepsilon, \delta)$ will first follow $\Gamma^{0}$ toward $\omega\left(N_{L}\right) \subset z$, then follow the trace of $\omega\left(N_{L}\right)$ in the vicinity of $\Lambda$ toward $\{\tau=1\}$, leave the vicinity of $z$, and, upon exiting, a portion of $M_{L}(\varepsilon, \delta)$ is $C^{1} O(\varepsilon)$-close to $W^{u}\left(\omega\left(N_{L}\right) \times(1-\rho, 1+\rho)\right)$ in the vicinity of $\Gamma^{1}$. Note that $\operatorname{dim} W^{u}\left(\omega\left(N_{L}\right) \times(1-\rho, 1+\rho)\right)=\operatorname{dim} M_{L}(\varepsilon, \delta)=4$.

It remains to show that $W^{u}\left(\omega\left(N_{L}\right) \times(1-\rho, 1+\rho)\right)$ intersects $M_{R}(\varepsilon)$ transversally since $M_{L}(\varepsilon, \delta)$ is $C^{1}$ $O(\varepsilon)$-close to $W^{u}\left(\omega\left(N_{L}\right) \times(1-\rho, 1+\rho)\right)$. Recall that, for $\varepsilon=0, M_{R}$ intersects $W^{u}(z)$ transversally along $N_{R}$ (Proposition 3.3); in particular, at $\gamma_{1}:=\alpha\left(\Gamma^{1}\right) \in \alpha\left(N_{R}\right) \subset Z$, we have

$$
T_{\gamma_{1}} M_{R}=T_{\gamma_{1}} \alpha\left(N_{R}\right) \oplus T_{\gamma_{1}} W^{u}\left(\gamma_{1}\right) \oplus \operatorname{span}\left\{V_{s}\right\},
$$

where, $T_{\gamma_{1}} W^{u}\left(\gamma_{1}\right)$ is the tangent space of the one-dimensional unstable fiber $W^{u}\left(\gamma_{1}\right)$ at $\gamma_{1}$ and the vector $V_{s} \notin T_{\gamma_{1}} W^{u}(Z)$ (the latter follows from the transversality of the intersection of $M_{R}$ and $W^{u}(Z)$ ). Also,

$$
T_{\gamma_{1}} W^{u}\left(\omega\left(N_{L}\right) \times(1-\rho, 1+\rho)\right)=T_{\gamma_{1}}\left(\omega\left(N_{L}\right) \cdot 1\right) \oplus \operatorname{span}\left\{V_{\tau}\right\} \oplus T_{\gamma_{1}} W^{u}\left(\gamma_{1}\right),
$$

where the vector $V_{\tau}$ is the tangent vector to the $\tau$-axis as a result of the interval factor $(1-\rho, 1+\rho)$. From Proposition 3.5, $\omega\left(N_{L}\right) \cdot 1$ and $\alpha\left(N_{R}\right)$ are transversal on $z \cap\{\tau=1\}$. Therefore, at $\gamma_{1}$, the tangent spaces 
$T_{\gamma_{1}} M_{R}$ and $T_{\gamma_{1}} W^{u}\left(\omega\left(N_{L}\right) \times(1-\rho, 1+\rho)\right)$ contain seven linearly independent vectors: $V_{s}, V_{\tau}, T_{\gamma_{1}} W^{u}\left(\gamma_{1}\right)$ and the other four from $T_{\gamma_{1}}\left(\omega\left(N_{L}\right) \cdot 1\right)$ and $T_{\gamma_{1}} \alpha\left(N_{R}\right)$; that is, $M_{R}$ and $W^{u}\left(\omega\left(N_{L}\right) \times(1-\rho, 1+\rho)\right)$ intersect transversally. We thus conclude that, there exists $0<\varepsilon_{0} \leq \varepsilon_{1}$ such that, if $0<\varepsilon \leq \varepsilon_{0}$, then $M_{L}(\varepsilon, \delta)$ intersects $M_{R}(\varepsilon)$ transversally.

For uniqueness, note that the transversality of the intersection $M_{L}(\varepsilon, \delta) \cap M_{R}(\varepsilon)$ implies $\operatorname{dim}\left(M_{L}(\varepsilon, \delta) \cap\right.$ $\left.M_{R}(\varepsilon)\right)=\operatorname{dim} M_{L}(\varepsilon, \delta)+\operatorname{dim} M_{R}(\varepsilon)-7=1$. Thus, there exists $\delta_{0}>0$ such that, if $0<\delta \leq \delta_{0}$, the intersection $M_{L}(\varepsilon, \delta) \cap M_{R}(\varepsilon)$ consists of precisely one solution near the singular orbit $\Gamma^{0} \cup \Lambda \cup \Gamma^{1}$.

\section{Qualitative properties of ionic flows without electroneutrality conditions}

Note that, under electroneutrality boundary conditions, two boundary layers disappear. Therefore, to examine the boundary layer effects on ionic flows, a first step is to remove the neutral conditions, more precisely, we assume

$$
-z_{2} L_{2}=\sigma\left(z_{1} L_{1}\right) \text { and }-z_{2} R_{2}=\rho\left(z_{1} R_{1}\right)
$$

for some positive constants $\sigma$ and $\rho$, which are not both equal to 1 ( $\sigma=1$ and $\rho=1$ in (4.1) implies electroneutrality conditions on boundary concentrations).

Recall from (3.10) that

$$
\begin{aligned}
& H(1) J_{1}(V ; 0)=\alpha_{0}\left(L_{1}, L_{2}, R_{1}, R_{2}\right)+\alpha_{1}\left(L_{1}, L_{2}, R_{1}, R_{2}\right) \frac{e}{k_{B} T} V, \\
& H(1) J_{2}(V ; 0)=\beta_{0}\left(L_{1}, L_{2}, R_{1}, R_{2}\right)+\beta_{1}\left(L_{1}, L_{2}, R_{1}, R_{2}\right) \frac{e}{k_{B} T} V
\end{aligned}
$$

where $H(1)=\int_{0}^{1} h^{-1}(s) d s$ and

$$
\begin{aligned}
& \alpha_{0}\left(L_{1}, L_{2}, R_{1}, R_{2}\right)=c_{1}^{L}-c_{1}^{R}+\frac{z_{1}\left(c_{1}^{L}-c_{1}^{R}\right) \ln \frac{L_{1} R_{2}}{L_{2} R_{1}}}{\left(z_{1}-z_{2}\right)\left(\ln c_{1}^{L}-\ln c_{1}^{R}\right)}, \\
& \alpha_{1}\left(L_{1}, L_{2}, R_{1}, R_{2}\right)=\frac{z_{1}\left(c_{1}^{L}-c_{1}^{R}\right)}{\ln c_{1}^{L}-\ln c_{1}^{R}}, \\
& \beta_{0}\left(L_{1}, L_{2}, R_{1}, R_{2}\right)=-\frac{z_{1}\left(c_{1}^{L}-c_{1}^{R}\right)}{z_{2}}-\frac{z_{1}\left(c_{1}^{L}-c_{1}^{R}\right) \ln \frac{L_{1} R_{2}}{L_{2} R_{1}}}{\left(z_{1}-z_{2}\right)\left(\ln c_{1}^{L}-\ln c_{1}^{R}\right)}, \\
& \beta_{1}\left(L_{1}, L_{2}, R_{1}, R_{2}\right)=-\frac{z_{1}\left(c_{1}^{L}-c_{1}^{R}\right)}{\ln c_{1}^{L}-\ln c_{1}^{R}},
\end{aligned}
$$

Notice that $c_{1}^{L}=\sigma^{\frac{z_{1}}{z_{1}-z_{2}}} L_{1}$ and $c_{1}^{R}=\rho^{\frac{z_{1}}{z_{1}-z_{2}}} R_{1}$. We rewrite (4.2) as

$$
\begin{aligned}
& \alpha_{0}(\sigma, \rho)=\sigma^{\frac{z_{1}}{z_{1}-z_{2}}} L_{1}-\rho^{\frac{z_{1}}{z_{1}-z_{2}}} R_{1}+\frac{z_{1}\left(\sigma^{\frac{z_{1}}{z_{1}-z_{2}}} L_{1}-\rho^{\frac{z_{1}}{z_{1}-z_{2}}} R_{1}\right) \ln \frac{\rho}{\sigma}}{\left(z_{1}-z_{2}\right)\left(\frac{z_{1}}{z_{1}-z_{2}} \ln \frac{\sigma}{\rho}+\ln L_{1}-\ln R_{1}\right)}, \\
& \alpha_{1}(\sigma, \rho)=\frac{z_{1}\left(\sigma^{\frac{z_{1}}{z_{1}-z_{2}}} L_{1}-\rho^{\frac{z_{1}}{z_{1}-z_{2}}} R_{1}\right)}{\frac{z_{1}}{z_{1}-z_{2}} \ln \frac{\sigma}{\rho}+\ln L_{1}-\ln R_{1}}, \\
& \beta_{0}(\sigma, \rho)=\frac{z_{1}\left(\sigma^{\frac{z_{1}}{z_{1}-z_{2}}} L_{1}-\rho^{\frac{z_{1}}{z_{1}-z_{2}}} R_{1}\right)}{-z_{2}}-\frac{z_{1}\left(\sigma^{\frac{z_{1}}{z_{1}-z_{2}}} L_{1}-\rho^{\frac{z_{1}}{z_{1}-z_{2}}} R_{1}\right) \ln \frac{\rho}{\sigma}}{\left(z_{1}-z_{2}\right)\left(\frac{z_{1}}{z_{1}-z_{2}} \ln \frac{\sigma}{\rho}+\ln L_{1}-\ln R_{1}\right)}, \\
& \beta_{1}(\sigma, \rho)=-\alpha_{1}(\sigma, \rho),
\end{aligned}
$$

Our main interest in this paper is to study the qualitative properties of individual fluxes and total flux defined in (2.3) close to the state of electroneutarlity, more precisely, under our setups, that is the case when 
$(\sigma, \rho) \rightarrow(1,1)$. To get started, we first expand $J_{k}$ at the point $\left(\sigma^{\star}, \rho^{\star}\right)=(1,1)$ up to the first order (we neglect higher order terms).

For convenience, we introduce functions $f_{0}=f_{0}\left(L_{1}, R_{1}\right), f_{1}=f_{1}\left(L_{1}, R_{1}\right), f_{2}=f_{2}\left(L_{1}, R_{1}\right)$ and $f_{3}=$ $f_{3}\left(L_{1}, R_{1}\right)$ given by

$$
\begin{aligned}
& f_{0}=L_{1}-\tilde{f}\left(L_{1}, R_{1}\right), f_{1}=R_{1}-\tilde{f}\left(L_{1}, R_{1}\right), \\
& f_{2}=-\frac{z_{1}}{z_{2}} L_{1}+\tilde{f}\left(L_{1}, R_{1}\right), f_{3}=\frac{z_{1}}{z_{2}} R_{1}-\tilde{f}\left(L_{1}, R_{1}\right),
\end{aligned}
$$

where

One can easily observe that

$$
\tilde{f}\left(L_{1}, R_{1}\right)=\frac{L_{1}-R_{1}}{\ln L_{1}-\ln R_{1}} .
$$

Proposition 4.1. Assume that $L_{1} \neq R_{1}$. One has

(i) $\tilde{f}\left(L_{1}, R_{1}\right)>0$.

(ii) $f_{0}\left(L_{1}, R_{1}\right)>0$ (resp. $\left.f_{1}\left(L_{1}, R_{1}\right)<0\right)$ for $L_{1}>R_{1}$, and $f_{0}\left(L_{1}, R_{1}\right)<0$ (resp. $\left.f_{1}\left(L_{1}, R_{1}\right)>0\right)$ for $L_{1}<R_{1}$.

(iii) $f_{2}\left(L_{1}, R_{1}\right)>0$ and $f_{3}\left(L_{1}, R_{1}\right)<0$.

In particular, as $L_{1} \rightarrow R_{1}, \tilde{f}\left(L_{1}, R_{1}\right) \rightarrow R_{1}>0, f_{0}\left(L_{1}, R_{1}\right) \rightarrow 0, f_{1}\left(L_{1}, R_{1}\right) \rightarrow 0, f_{2}\left(L_{1}, R_{1}\right) \rightarrow \frac{z_{1}-z_{1}}{z_{2}} R_{1}>0$, and $f_{3}\left(L_{1}, R_{1}\right) \rightarrow \frac{z_{1}-z_{2}}{z_{2}} R_{1}<0$.

Together with (4.4), system (4.3) can be written as (neglecting higher order terms in both $\sigma$ and $\rho$ )

$$
\begin{aligned}
& \alpha_{0}(\sigma, \rho)=\frac{z_{1}}{z_{1}-z_{2}}\left(f_{0}\left(L_{1}, R_{1}\right) \sigma-f_{1}\left(L_{1}, R_{1}\right) \rho\right)-\frac{z_{2}}{z_{1}-z_{2}}\left(L_{1}-R_{1}\right), \\
& \alpha_{1}(\sigma, \rho)=\frac{z_{1}^{2}}{\left(z_{1}-z_{2}\right)\left(\ln L_{1}-\ln R_{1}\right)}\left(f_{0}\left(L_{1}, R_{1}\right) \sigma-f_{1}\left(L_{1}, R_{1}\right) \rho\right)-\frac{z_{1} z_{2}}{z_{1}-z_{2}} \tilde{f}\left(L_{1}, R_{1}\right), \\
& \beta_{0}(\sigma, \rho)=\frac{z_{1}}{z_{1}-z_{2}}\left(f_{2}\left(L_{1}, R_{1}\right) \sigma+f_{3}\left(L_{1}, R_{1}\right) \rho+\left(L_{1}-R_{1}\right)\right), \\
& \beta_{1}(\sigma, \rho)=-\alpha_{1}(\sigma, \rho) .
\end{aligned}
$$

We use $J_{k}^{E N}$ (resp. $I^{E N}$ ) to denote the individual flux (resp. the total flux of charge) with the assumption of electroneutrality boundary conditions, and $J_{k}$ (resp. $I$ ) to denote the individual flux (resp. the total flux of charge) without the assumption of electroneutrality boundary conditions. To investigate the boundary layer effect on ionic flows, which is equivalent to the effects from the violation of electroneutrality boundary concentrations under our setups, we define three functions $\mathcal{D}_{1}(V ; \sigma, \rho)=\mathcal{D}_{1}\left(V ; \sigma, \rho ; L_{1}, R_{1}\right), \mathcal{D}_{2}(V ; \sigma, \rho)=$ $\mathcal{D}_{2}\left(V ; \sigma, \rho ; L_{1}, R_{1}\right)$ and $\mathcal{D}_{t}(V ; \sigma, \rho)=\mathcal{D}_{t}\left(V ; \sigma, \rho ; L_{1}, R_{1}\right)$ as follows:

$$
\begin{aligned}
H(1) \mathcal{D}_{1}(V ; \sigma, \rho)= & J_{1}\left(V ; L_{1}, R_{1} ; \sigma, \rho\right)-J_{1}^{E N}\left(V ; L_{1}, R_{1} ; \sigma, \rho\right) \\
= & \frac{z_{1}}{z_{1}-z_{2}}\left(1+\frac{e}{k_{B} T} \frac{z_{1} V}{\ln L_{1}-\ln R_{1}}\right) g_{1}\left(\sigma, \rho ; L_{1}, R_{1}\right), \\
H(1) \mathcal{D}_{2}(V ; \sigma, \rho)= & J_{2}\left(V ; L_{1}, R_{1} ; \sigma, \rho\right)-J_{2}^{E N}\left(V ; L_{1}, R_{1} ; \sigma, \rho\right) \\
= & \frac{z_{1}}{z_{1}-z_{2}}\left\{g_{2}\left(\sigma, \rho ; L_{1}, R_{1}\right)-\frac{e}{k_{B} T} \frac{z_{1} g_{1}\left(\sigma, \rho ; L_{1}, R_{1}\right) V}{\ln L_{1}-\ln R_{1}}\right\}, \\
H(1) \mathcal{D}_{t}(V ; \sigma, \rho)= & I\left(V ; L_{1}, R_{1} ; \sigma, \rho\right)-I^{E N}\left(V ; L_{1}, R_{1} ; \sigma, \rho\right) \\
= & \frac{z_{1}}{z_{1}-z_{2}}\left[z_{1} D_{1} g_{1}\left(\sigma, \rho ; L_{1}, R_{1}\right)-z_{2} D_{2} g_{2}\left(\sigma, \rho ; L_{1}, R_{1}\right)\right. \\
& \left.+\frac{e}{k_{B} T} \frac{z_{1}\left(z_{1} D_{1}+z_{2} D_{2}\right) g_{1}\left(\sigma, \rho ; L_{1}, R_{1}\right) V}{\ln L_{1}-\ln R_{1}}\right],
\end{aligned}
$$

where

$$
\begin{aligned}
& g_{1}\left(\sigma, \rho ; L_{1}, R_{1}\right)=f_{0}\left(L_{1}, R_{1}\right) \sigma-f_{1}\left(L_{1}, R_{1}\right) \rho-\left(L_{1}-R_{1}\right) \\
& g_{2}\left(\sigma, \rho ; L_{1}, R_{1}\right)=\frac{z_{1}}{z_{2}}\left((\rho-1) R_{1}+(1-\sigma) L_{1}\right)+(\sigma-\rho) \tilde{f}\left(L_{1}, R_{1}\right)
\end{aligned}
$$


For the function $g_{1}\left(\sigma, \rho ; L_{1}, R_{1}\right)$ defined in (4.6), one has

Lemma 4.2. Assume that $L_{1}>R_{1}$. One has

(i) $g_{1}\left(L_{1}, R_{1} ; \sigma, \rho\right)>0$ if either $(\sigma, \rho) \rightarrow\left(1^{+}, 1^{+}\right)$or $(\sigma, \rho) \rightarrow\left(1^{+}, 1^{-}\right)$with $\sigma+\rho>2$;

(ii) $g_{1}\left(L_{1}, R_{1} ; \sigma, \rho\right)<0$ if either $(\sigma, \rho) \rightarrow\left(1^{-}, 1^{-}\right)$or $(\sigma, \rho) \rightarrow\left(1^{-}, 1^{+}\right)$with $\sigma+\rho<2$;

(iii) There exists a unique $\tilde{x}_{1}>1$ such that for $(\sigma, \rho) \rightarrow\left(1^{-}, 1^{+}\right)$with $\sigma+\rho>2$, one has $g_{1}\left(L_{1}, R_{1} ; \sigma, \rho\right)<0$ if $\frac{L_{1}}{R_{1}}>\tilde{x}_{1}$ and $g_{1}\left(L_{1}, R_{1} ; \sigma, \rho\right)>0$ if $1<\frac{L_{1}}{R_{1}}<\tilde{x}_{1}$. In particular, $g_{1}\left(L_{1}, R_{1} ; \sigma, \rho\right)=0$ at $\frac{L_{1}}{R_{1}}=\tilde{x}_{1}$, where $\tilde{x}_{1}$ is the root of

$$
h_{1}(x)=(\sigma-1) x \ln x+(\rho-\sigma)(x-1)+(1-\rho) \ln x
$$

with $x$ being defined as $x=\frac{L_{1}}{R_{1}}$.

(iv) There exists a unique $\tilde{x}_{2}>1$ such that for $(\sigma, \rho) \rightarrow\left(1^{+}, 1^{-}\right)$with $\sigma+\rho<2$, one has $g_{1}\left(L_{1}, R_{1} ; \sigma, \rho\right)<0$ if $1<\frac{L_{1}}{R_{1}}<\tilde{x}_{2}$ and $g_{1}\left(L_{1}, R_{1} ; \sigma, \rho\right)>0$ if $\frac{L_{1}}{R_{1}}>\tilde{x}_{2}$. In particular, $g_{1}\left(L_{1}, R_{1} ; \sigma, \rho\right)=0$ at $\frac{L_{1}}{R_{1}}=\tilde{x}_{2}$, where $\tilde{x}_{2}$ is the root of

$$
h_{1}(x)=(\sigma-1) x \ln x+(\rho-\sigma)(x-1)+(1-\rho) \ln x
$$

with $x$ being defined as $x=\frac{L_{1}}{R_{1}}$.

Proof. Rewrite $g_{1}$ as $g_{1}\left(\sigma, \rho ; L_{1}, R_{1}\right)=R_{1} h(x)$ where, with $x=\frac{L_{1}}{R_{1}}>1$,

$$
h(x)=(\sigma-1) x+(\rho-\sigma) \frac{x-1}{\ln x}+1-\rho=\frac{h_{1}(x)}{\ln x},
$$

where

$$
h_{1}(x)=(\sigma-1) x \ln x+(\rho-\sigma)(x-1)+(1-\rho) \ln x .
$$

Note that $h_{1}(1)=0$,

$$
\begin{gathered}
h_{1}^{\prime}(x)=(\sigma-1) \ln x+\sigma-1+\rho-\sigma+\frac{1-\rho}{x} \Rightarrow h_{1}^{\prime}(1)=0 . \\
h_{1}^{\prime \prime}(x)=\frac{\sigma-1}{x}-\frac{1-\rho}{x^{2}}=\frac{(\sigma-1) x-(1-\rho)}{x^{2}} \Longrightarrow h_{1}^{\prime \prime}(x)=0 \Longleftrightarrow x^{*}=\frac{1-\rho}{\sigma-1},
\end{gathered}
$$

from which one has the following four cases

(A1) $\sigma>1$ and $\rho>1$, which implies that $h_{1}^{\prime \prime}(x)>0$ for all $x>1$. It follows that $h_{1}^{\prime}(x)$ is increasing for all $x>1$. Together with $h^{\prime}(1)=0$, we have $h^{\prime}(x)>0$ for all $x>1$, which implies that $h_{1}(x)$ is increasing for all $x>1$. Note that $h_{1}(1)=0$, one can conclude that $h_{1}(x)>0$ for all $x>1$. Thus, $h(x)>0$ for all $x>1$, and hence $g_{1}\left(L_{1}, R_{1} ; \sigma, \rho\right)>0$ for $L_{1}>R_{1}$.

(A2) $0<\sigma<1$ and $0<\rho<1$, which implies that $h_{1}^{\prime \prime}(x)<0$ for all $x>1$. A similar argument as in case (A1) gives $g_{1}\left(L_{1}, R_{1} ; \sigma, \rho\right)<0$ for $L_{1}>R_{1}$.

(A3) $0<\sigma<1$ and $\rho>1$, which implies that $h_{1}^{\prime \prime}(x)>0$ if $x<x^{\star}$, and $h_{1}^{\prime \prime}(x)<0$ if $x>x^{\star}$. We have the following two subcases

(A31) $\sigma+\rho>2$, which implies that $x^{\star}>1$. For this subcase, there exists another critical value $\bar{x}_{1}>x^{\star}$ of $h_{1}(x)$ such that $h_{1}^{\prime}(x)>0$ for $1<x<\bar{x}_{1}$ and $h_{1}^{\prime}(x)<0$ for $x>\bar{x}_{1}$. Note that $h_{1}(1)=0$ and $h_{1}(\infty)=-\infty$. There exists $\tilde{x}_{1}>\bar{x}_{1}$ such that $h_{1}(x)>0$ for $1<x<\tilde{x}_{1}$, and $h_{1}(x)<0$ for $x>\tilde{x}_{1}$. Therefore, $g_{1}\left(L_{1}, R_{1} ; \sigma, \rho\right)>0$ for $1<\frac{L_{1}}{R_{1}}<\tilde{x}_{1}$, and $g_{1}\left(L_{1}, R_{1} ; \sigma, \rho\right)<0$ for $\frac{L_{1}}{R_{1}}>\tilde{x}_{1}$.

(A32) $\sigma+\rho<2$, which implies that $0<\chi^{\star}<1$. A direct argument leads to $g_{1}\left(L_{1}, R_{1} ; \sigma, \rho\right)<0$ for $L_{1}>R_{1}$.

(A4) $\sigma>1$ and $0<\rho<1$, which implies $h_{1}^{\prime \prime}(x)>0$ if $x>x^{\star}$, and $h_{1}^{\prime \prime}(x)<0$ if $x<x^{\star}$.

(A41) $\sigma+\rho>2$, which implies that $0<x^{\star}<1$. For this subcase, one has $g_{1}\left(L_{1}, R_{1} ; \sigma, \rho\right)>0$ for $L_{1}>R_{1}$.

(A42) $\sigma+\rho<2$, which implies that $x^{\star}>1$. There exists another critical value $\bar{x}_{2}>x^{\star}$ of $h_{1}(x)$ such that $h_{1}^{\prime}(x)<0$ for $1<x<\bar{x}_{2}$ and $h_{1}^{\prime}(x)>0$ for $x>\bar{x}_{2}$. Note that $h_{1}(1)=0$ and $h_{1}(\infty)=\infty$. There exists $\tilde{x}_{2}>\bar{x}_{2}$ such that $h_{1}(x)<0$ for $1<x<\tilde{x}_{2}$, and $h_{1}(x)>0$ for $x>\tilde{x}_{2}$. Therefore, $g_{1}\left(L_{1}, R_{1} ; \sigma, \rho\right)<0$ for $1<\frac{L_{1}}{R_{1}}<\tilde{x}_{2}$, and $g_{1}\left(L_{1}, R_{1} ; \sigma, \rho\right)>0$ for $\frac{L_{1}}{R_{1}}>\tilde{x}_{2}$. 
This completes the proof.

We assume that $g_{1}\left(V ; \sigma, \rho ; L_{1}, R_{1}\right)$ defined in (4.6) is not zero, and identify three critical potentials $V_{1 c}, V_{2 c}$ and $V_{c}$ by

$$
\mathcal{D}_{1}\left(V_{1 c} ; \sigma, \rho ; L_{1}, R_{1}\right)=0, \mathcal{D}_{2}\left(V_{2 c} ; \sigma, \rho ; L_{1}, R_{1}\right)=0 \text { and } \mathcal{D}_{t}\left(V_{c} ; \sigma, \rho ; L_{1}, R_{1}\right)=0 \text {, }
$$

which are given by

$$
\begin{aligned}
V_{1 c} & =\frac{k_{B} T\left(\ln L_{1}-\ln R_{1}\right)}{z_{1} e}, \\
V_{2 c} & =\frac{k_{B} T\left(\ln L_{1}-\ln R_{1}\right)}{z_{1} e} \cdot \frac{g_{2}\left(\sigma, \rho ; L_{1}, R_{1}\right)}{g_{1}\left(\sigma, \rho ; L_{1}, R_{1}\right)}, \\
V_{c} & =\frac{k_{B} T\left(\ln L_{1}-\ln R_{1}\right)}{z_{1} e\left(z_{1} D_{1}+z_{2} D_{2}\right)} \cdot \frac{z_{1} D_{1} g_{1}\left(L_{1}, R_{1} ; \sigma, \rho\right)-z_{2} D_{2} g_{2}\left(\sigma, \rho ; L_{1}, R_{1}\right)}{g_{1}\left(\sigma, \rho ; L_{1}, R_{1}\right)} .
\end{aligned}
$$

From Lemma 4.2, together with $H(1)=\int_{0}^{1} h^{-1}(s) d s>0$ and the critical potentials identified in (4.7), we obtain

Theorem 4.3. Assume $L_{1}>R_{1}$ and $z_{1} D_{1}>-z_{2} D_{2}$. For small $\varepsilon>0$, as $(\sigma, \rho) \rightarrow\left(1^{+}, 1^{+}\right)$, one has

(i) $\mathcal{D}_{1}(V)>0\left(\right.$ resp. $\left.\mathcal{D}_{1}(V)<0\right)$ for $V>V_{1 c}\left(\right.$ resp. $\left.V<V_{1 c}\right)$. Furthermore, $\mathcal{D}_{1}(V)$ is increasing in the potential $V$, that is, the boundary layer effects on the individual flux $J_{1}$ becomes stronger as $V>V_{1 c}$ becomes larger.

(ii) $\mathcal{D}_{2}(V)>0\left(\right.$ resp. $\left.\mathcal{D}_{2}(V)<0\right)$ for $V<V_{2 c}$ (resp. $V>V_{2 c}$ ). Furthermore, $\mathcal{D}_{2}(V)$ is decreasing in the potential $V$, that is, the boundary layer effects on the individual flux $J_{2}$ becomes weaker as $V>V_{2 c}$ becomes larger.

(iii) $\mathcal{D}_{t}>0\left(\right.$ resp. $\left.\mathcal{D}_{t}<0\right)$ for $V>V_{c}$ (resp. $\left.V<V_{c}\right)$. Furthermore, $\mathcal{D}_{2}(V)$ is increasing in the potential $V$, that is, the boundary layer effects on the total flux I becomes stronger as $V>V_{c}$ becomes larger.

Theorem 4.4. Assume $L_{1}>R_{1}$ and $z_{1} D_{1}>-z_{2} D_{2}$. For small $\varepsilon>0$, as $(\sigma, \rho) \rightarrow\left(1^{-}, 1^{-}\right)$, one has

(i) $\mathcal{D}_{1}(V)>0\left(\right.$ resp. $\left.\mathcal{D}_{1}(V)<0\right)$ for $V<V_{1 c}\left(\right.$ resp. $\left.V>V_{1 c}\right)$. Furthermore, $\mathcal{D}_{1}(V)$ is decreasing in the potential $V$, that is, the boundary layer effects on the individual flux $J_{1}$ becomes weaker as $V>V_{1 c}$ becomes larger.

(ii) $\mathcal{D}_{2}(V)>0\left(\right.$ resp. $\left.\mathcal{D}_{2}(V)<0\right)$ for $V>V_{2 c}\left(\right.$ resp. $\left.V<V_{2 c}\right)$. Furthermore, $\mathcal{D}_{2}(V)$ is increasing in the potential $V$, that is, the boundary layer effects on the individual flux $J_{2}$ becomes stronger as $V>V_{2 c}$ becomes larger.

(iii) $\left.\mathcal{D}_{t}\right\rangle 0$ (resp. $\left.\mathcal{D}_{t}<0\right)$ for $V\left\langle V_{c}(\right.$ resp. $\left.V\rangle V_{c}\right)$. Furthermore, $\mathcal{D}_{2}(V)$ is decreasing in the potential $V$, that is, the boundary layer effects on the total flux I becomes weaker as $V>V_{c}$ becomes larger.

Remark 4.5. Similar results can be obtained for other cases stated in Lemma 4.2.

\section{Concluding Remarks}

In this work, we consider the classical PNP model to study the boundary layer effects on ionic flows for two ion species, one positively charged and one negatively charged. Mathematically, we examine the qualitative properties of ionic flows, in terms of both individual fluxes $J_{k}$ and the total flux I without the assumption of electroneutrality conditions, which is more realistic in the study of ion channel problems. It turns out that the flow properties of interest depend very sensitively on boundary concentrations (in terms of $\left(L_{1}, R_{1}\right)$ ) in addition to other physical parameters such as boundary potentials, ion valences and diffusion coefficients. For the relatively simple setting and assumptions of the model studied in this paper, we are able to characterize the distinct effects of the nonlinear interplay between these physical parameters. The dynamical behaviors of ionic flows studied in this work are much more rich compared to previous studied under the assumption of electroneutrality conditions. Our study also provides a nice way to adjust the ionic flows through membrane channels by controlling boundary potentials for fixed boundary concentrations. 
We finally comment that the model studied in this paper is oversimplified and the specific setting (such as zero permanent charge assumption) of our problem may not reflect precisely any realistic biological settings. However, we believe the existence of these critical potentials are generally in valid (even for PNP model with nonzero permanent charge, and the awareness of the potential existence of these critical voltage itself would be useful for further numerical studies and stimulate further analytical studies of ionic flows through ion channels.

Acknowledgement: M. Zhang was partially supported by AMS-Simon Travel Grant.

\section{References}

[1] N. Abaid, R. S. Eisenberg, and W. Liu, Asymptotic expansions of I-V relations via a Poisson-Nernst-Planck system. SIAM J. Appl. Dyn. Syst., 7 (2008), 1507-1526.

[2] V. Barcilon, Ion flow through narrow membrane channels: Part I. SIAM J. Appl. Math., 52 (1992), 1391-1404.

[3] V. Barcilon, D. -P. Chen, and R. S. Eisenberg, lon flow through narrow membrane channels: Part II. SIAM J. Appl. Math., 52 (1992), 1405-1425.

[4] V. Barcilon, D.-P. Chen, R. S. Eisenberg, and J. W. Jerome, Qualitative properties of steady-state Poisson-Nernst-Planck systems: Perturbation and simulation study. SIAM J. Appl. Math., 57 (1997), 631-648.

[5] P. W. Bates, Y. Jia, G. Lin, H. Lu, and M. Zhang: Individual flux study via steady-state Poisson-Nernst-Planck systems: Effects from boundary conditions. SIAM J. Appl. Dyn. Syst., 16 (2017), 410-430.

[6] P. W. Bates, W. Liu, H. Lu and M. Zhang, Ion size and valence effects on ionic flows via Poisson-Nernst-Planck models. Commu. Math. Sci., 15 (2017), 881-901.

[7] D. Boda, D. Busath, B. Eisenberg, D. Henderson, and W. Nonner, Monte Carlo simulations of ion selectivity in a biological $\mathrm{Na}^{+}$channel: charge-space competition. Phys. Chem. Chem. Phys., 4 (2002), 5154-5160.

[8] D. Boda, D. Gillespie, W. Nonner, D. Henderson, and B. Eisenberg, Computing induced charges in inhomogeneous dielectric media: application in a Monte Carlo simulation of complex ionic systems. Phys. Rev. E, 69 (2004), 1-10.

[9] M. Burger, R. S. Eisenberg, and H. W. Engl, Inverse problems related to ion channel selectivity. SIAM J. Appl. Math., 67 (2007), 960-989.

[10] A. E. Cardenas, R. D. Coalson, and M. G. Kurnikova, Three-Dimensional Poisson-Nernst-Planck Theory Studies: Influence of Membrane Electrostatics on Gramicidin A Channel Conductance. Biophys. J., 79 (2000), 80-93.

[11] D. Chen, A New Poisson-Nernst-Planck Model with Ion-Water Interactions for Charge Transport in lon Channels. Bull Math Biol., 78 (2016), 1703-1726.

[12] D. P. Chen and R. S. Eisenberg, Charges, currents and potentials in ionic channels of one conformation. Biophys. J., 64 (1993), 1405-1421.

[13] S. Chung and S. Kuyucak, Predicting channel function from channel structure using Brownian dynamics simulations. Clin. Exp. Pharmacol Physiol., 28 (2001), 89-94.

[14] R. Coalson and M. Kurnikova, Poisson-Nernst-Planck theory approach to the calculation of current through biological ion channels. IEEE Transaction on NanoBioscience, 4 (2005), 81-93.

[15] B. Eisenberg, Ion Channels as Devices. J. Comp. Electro., 2 (2003), 245-249.

[16] B. Eisenberg, Proteins, Channels, and Crowded lons. Biophys. Chem., 100 (2003), 507 - 517.

[17] R. S. Eisenberg, Channels as enzymes. J. Memb. Biol., 115 (1990), 1-12.

[18] R. S. Eisenberg, Atomic Biology, Electrostatics and Ionic Channels. In New Developments and Theoretical Studies of Proteins, R. Elber, Editor, 269-357, World Scientific, Philadelphia, 1996.

[19] R. S. Eisenberg, From Structure to Function in Open Ionic Channels. J. Memb. Biol., 171 (1999), 1-24.

[20] B. Eisenberg and W. Liu, Poisson-Nernst-Planck systems for ion channels with permanent charges. SIAM J. Math. Anal., 38 (2007), 1932-1966.

[21] D. Gillespie, A singular perturbation analysis of the Poisson-Nernst-Planck system: Applications to lonic Channels. Ph. $D$ Dissertation, Rush University at Chicago, (1999).

[22] D. Gillespie and R. S. Eisenberg, Physical descriptions of experimental selectivity measurements in ion channels. European Biophys. J., 31 (2002), 454-466.

[23] D. Gillespie, W. Nonner, and R. S. Eisenberg, Coupling Poisson-Nernst-Planck and density functional theory to calculate ion flux. J. Phys.: Condens. Matter, 14 (2002), 12129-12145.

[24] D. Gillespie, W. Nonner, and R. S. Eisenberg, Density functional theory of charged, hard-sphere fluids. Phys. Rev. E, 68 (2003), 1-10.

[25] D. Gillespie, W. Nonner, and R. S. Eisenberg, Crowded Charge in Biological Ion Channels. Nanotech., 3 (2003), 435-438. 
[26] U. Hollerbach, D. Chen, W. Nonner, and B. Eisenberg, Three-dimensional Poisson-Nernst-Planck Theory of Open Channels. Biophys. J. , 76 (1999), p. A205.

[27] Y. Hyon, B. Eisenberg, and C. Liu, A mathematical model for the hard sphere repulsion in ionic solutions. Commun. Math. Sci., 9 (2010), 459-475.

[28] Y. Hyon, J. Fonseca, B. Eisenberg, and C. Liu, Energy variational approach to study charge inversion (layering) near charged walls. Discrete Contin. Dyn. Syst. Ser. B, 17 (2012), 2725-2743.

[29] Y. Hyon, C. Liu, and B. Eisenberg, PNP equations with steric effects: a model of ion flow through channels. J. Phys. Chem. B, 116 (2012), 11422-11441.

[30] W. Im, D. Beglov, and B. Roux, Continuum solvation model: Electrostatic forces from numerical solutions to the PoissonBolztmann equation. Comp. Phys. Comm., 111 (1998), 59-75.

[31] W. Im and B. Roux, Ion permeation and selectivity of OmpF porin: a theoretical study based on molecular dynamics, Brownian dynamics, and continuum electrodiffusion theory. J. Mol. Biol., 322 (2002), 851-869.

[32] S. Ji and W. Liu, Poisson-Nernst-Planck Systems for Ion Flow with Density Functional Theory for Hard-Sphere Potential: I-V relations and Critical Potentials. Part I: Analysis. J. Dyn. Diff. Equat., 24 (2012), 955-983.

[33] S. Ji, W. Liu and M. Zhang, Effects of (small) permanent charges and channel geometry on ionic flows via classical PoissonNernst-Planck models. SIAM J. on Appl. Math., 75 (2015), 114-135.

[34] Y. Jia, W. Liu and M. Zhang, Qualitative properties of ionic flows via Poisson-Nernst-Planck systems with Bikerman's local hard-sphere potential: Ion size effects. Discrete and Continuous Dynamical Systems-B, 21 (2016), 1775-1802.

[35] C. Jones, Geometric singular perturbation theory. Dynamical systems (Montecatini Terme, 1994), pp. 44-118. Lect. Notes in Math. 1609, Springer, Berlin, 1995.

[36] C. Jones, T. Kaper, and N. Kopell, Tracking invariant manifolds up tp exponentially small errors. SIAM J. Math. Anal., 27 (1996), 558-577.

[37] C. Jones and N. Kopell, Tracking invariant manifolds with differential forms in singularly perturbed systems. J. Differential Equations, 108 (1994), 64-88.

[38] M. S. Kilic, M. Z. Bazant, and A. Ajdari, Steric effects in the dynamics of electrolytes at large applied voltages. II. Modified Poisson-Nernst-Planck equations. Phys. Rev. E, 75 (2007), 021503.

[39] C.-C. Lee, H. Lee, Y. Hyon, T.-C. Lin, and C. Liu, New Poisson-Boltzmann type equations: one-dimensional solutions. Nonlinearity, 24 (2011), 431-458.

[40] G. Lin, W. Liu, Y. Yi and M. Zhang: Poisson-Nernst-Planck systems for ion flow with density functional theory for local hardsphere potential. SIAM J. Appl. Dyn. Syst., 12 (2013), 1613-1648.

[41] W. Liu, Geometric singular perturbation approach to steady-state Poisson-Nernst-Planck systems. SIAM J. Appl. Math., 65 (2005), 754-766.

[42] W. Liu, One-dimensional steady-state Poisson-Nernst-Planck systems for ion channels with multiple ion species. J. Differential Equations, 246 (2009), 428-451.

[43] W. Liu and H. Xu, A complete analysis of a classical Poisson-Nernst-Planck model for ionic flow, J. Differential Equations, 258 (2015), 1192-1228.

[44] W. Liu and B. Wang, Poisson-Nernst-Planck systems for narrow tubular-like membrane channels. J. Dyn. Diff. Equat., 22 (2010), 413-437.

[45] W. Liu, X. Tu, and M. Zhang, Poisson-Nernst-Planck Systems for Ion Flow with Density Functional Theory for Hard-Sphere Potential: I-V relations and Critical Potentials. Part II: Numerics. J. Dyn. Diff. Equat., 24 (2012), 985-1004.

[46] B. Nadler, Z. Schuss, A. Singer, and B. Eisenberg, Diffusion through protein channels: from molecular description to continuum equations. Nanotech., 3 (2003), 439-442.

[47] W. Nonner, L. Catacuzzeno, and B. Eisenberg, Binding and selectivity on L-type Ca channels: a mean spherical approximation. Biophys. J., 79 (2000), 1976-1992.

[48] W. Nonner, D.-P. Chen, and B. Eisenberg, Anomalous mole fraction effect, electrostatics, and binding in ionic channels, Biophys. J., 74 (1998), pp. 2327?2334.

[49] W. Nonner, D. Gillespie, D. Henderson, and B. Eisenberg, Ion accumulation in a biological calcium channel: Effects of solvent and confining pressure, J. Phys. Chem. B, 105 (2001), 6427-6436.

[50] W. Nonner, A. Peyser, D. Gillespie, and B. Eisenberg, Relating microscopic charge movement to macroscopic currents: The Ramo-Shockley theorem applied to ion channels, Biophys. J., 87 (2004), 3716-3722.

[51] W. Nonner and R. S. Eisenberg, Ion permeation and glutamate residues linked by Poisson-Nernst-Planck theory in L-type Calcium channels. Biophys. J., 75 (1998), 1287-1305.

[52] S. Y. Noskov, W. Im, and B. Roux, Ion Permeation through the $z_{1}$-Hemolysin Channel: Theoretical Studies Based on Brownian Dynamics and Poisson-Nernst-Planck Electrodiffusion Theory. Biophys. J., 87 (2004), 2299-2309.

[53] J.-K. Park and J. W. Jerome, Qualitative properties of steady-state Poisson-Nernst-Planck systems: Mathematical study. SIAM J. Appl. Math., 57 (1997), 609-630.

[54] B. Roux, T. W. Allen, S. Berneche, and W. Im, Theoretical and computational models of biological ion channels. Quat. Rev. Biophys., 37 (2004), 15-103.

[55] Z. Schuss, B. Nadler, and R. S. Eisenberg, Derivation of Poisson and Nernst-Planck equations in a bath and channel from a molecular model. Phys. Rev. E, 64 (2001), 1-14. 
[56] A. Singer, D. Gillespie, J. Norbury, and R. S. Eisenberg, Singular perturbation analysis of the steady-state Poisson-NernstPlanck system: applications to ion channels. European J. Appl. Math., 19 (2008), 541-560.

[57] A. Singer and J. Norbury, A Poisson-Nernst-Planck model for biological ion channels-an asymptotic analysis in a threedimensional narrow funnel. SIAM J. Appl. Math., 70 (2009), 949-968.

[58] S.-K. Tin, N. Kopell, and C. Jones, Invariant manifolds and singularly perturbed boundary value problems. SIAM J. Numer. Anal., 31 (1994), 1558-1576.

[59] X.-S. Wang, D. He, J. Wylie, and H. Huang, Singular perturbation solutions of steady-state Poisson-Nernst-Planck systems. Phys. Rev. E, 89 (2014), 1-14.

[60] G. W. Wei, Q. Zheng, Z. Chen, and K. Xia, Variational multiscale models for charge transport. SIAM Review, 54 (2012), 699 754.

[61] M. Zhang, Asymptotic expansions and numerical simulations of I-V relations via a steady-state Poisson-Nernst-Planck system. Rocky Mountain J. Math., 45 (2015), 1681-1708.

[62] J. Zhang, D. Acheampong and M. Zhang: Effects on ionic flows from finite ion sizes via Poisson-Nernst-Planck models with non-local excess chemical potentials. Molecular Based Math. Biology, 5 (2017), 58-77.

[63] Q. Zheng and G. W. Wei, Poisson-Boltzmann-Nernst-Planck model. J. Chem. Phys., 134 (2011), 194101 (1-17). 